\title{
Publicidad on-line y Nuevas Tecnologías de Comunicación: el Anunciante Franquiciador
}

Juan Monserrat-Gauchi | Juan.Monserrat@ua.es

David Blázquez Álvarez I David.Blazquez@ua.es

Universidad de ALICANTE

Resumen: La Publicidad en el medio Internet y las Nuevas Tecnologías de Comunicación conllevan un cambio sustancial en la forma de relacionarse del anunciante con sus públicos. El anunciante pierde su protagonismo y el rol de "emisor" en la comunicación comercial. La morfogénesis de los sistemas de comunicación es una realidad constatada.

Palabras clave: Comunicación, Publicidad, Nuevas-Tecnologías, Franquicia

Abstract: Advertising in the context of the Internet and new information and communication technologies means a substantial change in the way advertisers interact with their target audiences. Advertisers are losing their leading roles as "addressers" in commercial communication. The process of morphogenesis in current communication systems is a verified fact in present times.

Key words: communication, advertising, new-technologies, franchising. 


\section{Introducción}

La aparición de las Nuevas Tecnologías de Información y Comunicación (NTIC) y su reciente auge ha supuesto un cambio importante en la gestión de la comunicación comercial en el seno de las empresas anunciantes. Este cambio afecta a todos los sujetos del tradicional Sistema Publicitario que, en estos últimos tiempos, sufre importantes reestructuraciones. (Caro, 2009; Campos, 2010; Hernández, 2010; Wallovis y Virgili, 2011).

Existen además, otros factores a tener en cuenta: como la crisis financiera mundial y la globalización de los mercados, además del auge de las nuevas tecnologías, que hacen que los tradicionales roles asignados a las empresas como anunciantes, a los medios como transmisores de sus órdenes y a los consumidores como público receptor pasivo de sus mensajes hayan cambiado (Buzzi, 2009).

La influencia de las NTIC, si bien cambia el paradigma publicitario y el rol de los principales agentes de este sistema, afecta más todavía a las empresas cuantos más públicos objetivo posean. La pérdida del control de la emisión de mensajes por parte del anunciante es un hecho. El público objetivo y los consumidores son emisores a la vez que receptores. Pero ¿qué ocurre en las empresas cuyos productos/servicios se comercializan mediante franquicia? El entramado comunicacional y la revolución que supone la irrupción de las NTIC es aún mayor ${ }^{1}$.

La franquicia es un sistema de comercialización de bienes y servicios que actualmente goza de un auge imparable. Como consecuencia de la globalización y de las ventajas de este sistema de comercialización, la fisionomía de las principales calles, centros y áreas comerciales no sólo de España, sino también de numerosos países de Europa, América y Asia, ha cambiado notablemente. Los porcentajes de comercio minorista integrado frente a los de comercio minorista independiente auguran un crecimiento que aún no ha tocado techo en muchos países: en Francia el $25 \%$ del comercio minorista opera integrado en alguna red, en Reino Unido es el 35\% y en España los minoristas integrados suponen el 15\%. En otros países como Estados Unidos el porcentaje está en torno al 50\%, casi la mitad del pequeño comercio opera integrado en alguno de los múltiples sistemas de comercio asociado.

Esta investigación parte de la base, ya demostrada, de que la comunicación en la empresa de franquicia fluye de forma diferente a las empresas convencionales, por lo que la gestión de las Nuevas Tecnologías de Información y Comunicación (NTIC) también plantea la necesidad de una gestión diferente, adecuada a cada uno de los públicos de la franquicia (Monserrat-Gauchi, J.:2005).

El estudio de las NTIC es necesario plantearlo desde la central de franquicia para cada uno de los tres principales públicos objetivo de la franquicia: los franquiciados actuales, los

\footnotetext{
${ }^{1}$ En este artículo se ofrecen los resultados de un Proyecto de Investigación Emergente financiado por el Vicerrectorado de Investigación de la Universidad de Alicante, cuya referencia es: GRE08-J02.
} 
franquiciados potenciales y los clientes finales de sus establecimientos. Esta es la peculiaridad de la franquicia a la que antes se ha hecho referencia: la existencia de un intermediario (el franquiciado) que supone nexo de unión entre la central o casa matriz y los clientes o consumidores finales del producto o servicio. El estudio de la utilización de estas tecnologías se realiza, pues, desde el conocimiento que desde la central franquiciadora se deberían aprovechar las NTIC para comunicarse con: los franquiciados actuales de la central, los clientes finales de sus establecimientos y con los inversores decididos a montar un establecimiento franquiciado.

Si se tiene en cuenta que cada establecimiento de la red, puede gestionar la comunicación con su propio público objetivo y entorno, este entramado comunicacional se complica todavía más.

\section{Estado de la Cuestión. Hipótesis y Objetivos de la Investigación}

El estudio del sistema de comercialización en franquicia se ha abordado desde diferentes perspectivas (Baena, 2010): legal, organización empresarial, marketing, management, estudios de satisfacción de empleados, rentabilidad de la distribución minorista independiente o mediante franquicia, etc. Sin embargo, son pocas las publicaciones de carácter científico que abordan el estudio de la franquicia en lo que respecta a la gestión de la comunicación. En este sentido, cabría destacar las aportaciones de Bolea de Anta (1988 y 1990) y Monserrat-Gauchi (2005 y 2008) en el ámbito nacional español, y en el ámbito anglosajón o americano de Dickey (2001), Davis (2004) o Lair (2006). Otros autores se acercan al estudio del sistema de franquicia al estudiar flujos de comunicación en diferentes sistemas de organización empresarial.

Desde hace años, la franquicia en España sigue un crecimiento casi continuado que se mantiene, incluso, en la presente época de crisis. Como indica Tormo, pese a los cierres que hubo durante 2008 (inicio de la actual crisis económica), al final del año estaban en marcha 63 enseñas más que en el ejercicio anterior, llegando a un total de 968 enseñas franquiciadoras con 69.081 establecimientos, lo que también supone un crecimiento de 700 locales. (Tormo, E. 2009)

Los datos aportados por Tormo coinciden aproximadamente con los resultados publicados en la guía editada por Barbadillo en 2009. Barbadillo indica concretamente un crecimiento del $18 \%$ en el número de enseñas en 2008 respecto a 2007, pero también advierte que el número de franquicias desaparecidas durante 2008, asciende a 200 enseñas, una cifra superior a la de años anteriores, en los cuales las bajas se cifraban en 100 ó 150.

Como indica Tormo en su Informe de la Franquicia en España, en el 2008 se creció un 2,8 por encima de la media del comercio minorista, y también por encima del PIB, siendo uno de los sectores que mejor está atravesando la presente crisis. No obstante, los datos del último año analizado, 2010, arrojan un crecimiento negativo en cuanto a la facturación global del sistema de franquicia, concretamente del $-0,2 \%$. El número de establecimientos operativos 
adheridos a una u otra red también ha descendido, reduciéndose en 695 locales menos repecto al año anterior, 2009.

Respecto a la importancia de la comunicación y a su contribución en el desarrollo y crecimiento de las franquicias en sus respectivas áreas de actuación, Mohr, Fisher y Nevir (1996), afirman que la comunicación es el nexo de unión fundamental entre los diferentes agentes del sistema. La comunicación favorece las relaciones que se generan en el sistema de franquicia (Anderson y Narus, 1984; Mohr y Nevin, 1990). De acuerdo con Anderson y Weitz, (1989) podemos afirmar que la franquicia responde a un modelo de negocio que exige una muy buena relación entre las partes $y$, en este sentido, la correcta gestión de la comunicación y la confianza entre franquiciador y franquiciado, son dos variables indispensables para evitar conflictos y consolidar relaciones. Algunos estudios han demostrado que existen ciertos aspectos que satisfacen al franquiciado, como puede ser el uso de marcas reconocidas o el envío de boletines de información y manuales (Knight, 1986), aspectos directamente relacionados con la comunicación franquiciador-franquiciado.

Son muchos los autores (Allen, 1994; Mubleman, 1996; Laurie, 2000; Tikoo, 2002; Monroy and Alzola, 2005) que afirman que la comunicación es la vía para conseguir una buena relación en el sistema de franquicia. Siguiendo con esta afirmación, los investigadores parten de la premisa de que es necesario gestionar la comunicación atendiendo, además, a los cambios tecnológicos en los que las empresas se desenvuelven.

Teniendo en cuenta los datos expuestos de crecimiento de empresas franquiciadoras en los últimos años, incuso en época de crisis económica, los investigadores se plantean la hipótesis de que la correcta gestión de la publicidad on-line y de las Nuevas Tecnologías de Información y Comunicación, es un hecho constatado en este tipo de anunciante, incluso en época de crisis.

Por todo ello, la hipótesis de partida de la presente investigación, sustentada desde el punto de vista teórico en el seguimiento y análisis de la bibliografía citada, quedaría planteada del siguiente modo: la gestión de las NTIC en las empresas franquiciadoras se lleva a cabo de forma integrada con el plan de marketing y comunicación de la empresa, creando estrategias de publicidad integrada que necesariamente articulen recursos publicitarios convencionales y NTIC.

Y para demostrar dicha hipótesis se plantean, dos objetivos a acometer. El primer objetivo es realizar un análisis descriptivo del uso de las NTIC por parte de las empresas durante el año 2009 y, el segundo, diagnosticar el uso de las NTC en dichas empresas.

El primer objetivo, plantea el análisis de tres usos complementarios, pero diferenciados, de las NTIC. Esos tres usos coinciden en con el triple público al que se dirige la empresa franquiciadora, se analizará el uso de las NTIC por parte de las enseñas para comunicarse 
con franquiciados en potencia, con los franquiciados actuales $y$, con los públicos objetivos finales.

No sólo se considera interesante describir las técnicas y herramientas utilizadas, sino también analizar qué agente realiza técnicamente esas acciones (si la propia empresa o una agencia de publicidad), la efectividad de las mismas y las distintas interrelaciones del uso de las NTIC con variables del tipo: número de franquicias, sector de actividad.

De este primer objetivo, al ser puramente descriptivo de un corte transversal temporal, se extrae otro subobjetivo de gran actualidad: mostrar como se están utilizando las NTIC en las franquicias en tiempos de crisis. Y para ello estudiaremos variables especialmente diseñadas ad hoc.

El segundo objetivo, el diagnóstico del uso de las NTIC en la empresa franquiciadora, pretende reflejar la situación actual respecto al uso de las herramientas informáticas aplicadas a la comunicación empresarial, contemplando si este uso está integrado en los planes de Marketing y Comunicación del a empresa y si forma parte de las estrategias definidas en los mismos. En definitiva, analizar la confluencia de las nuevas "redes" digitales con los medios tradicionales y conocer la integración de las NTIC en los planes de comunicación de la empresa.

\section{Metodología}

En el presente trabajo, los autores han utilizado diversas herramientas de investigación científica para aportar rigor a un estudio enmarcado dentro de las llamadas Ciencias Sociales.

En esta investigación se han utilizado tanto técnicas cualitativas, como cuantitativas, ambas para conseguir los mismos fines y objetivos. A continuación, se detalla el desarrollo del Panel de expertos para la configuración de la herramienta metodológica de análisis (cuestionario), mediante la consulta y reunión de expertos del ámbito de la investigación. Una vez definido el cuestionario, se procedió a instar a las empresas franquiciadoras de nuestra muestra de estudio, a que lo respondieran. $\mathrm{Y}$ con el tratamiento estadístico de las respuestas a ese cuestionario se obtuvieron los resultados que se presentan en el siguiente epígrafe.

El proceso de trabajo seguido en esta investigación puede resumirse de la siguiente forma:

\subsection{Revisión Bibliográfica}

La investigación que nos ocupa se apoyó en la observación documental de dos tipos. En el primero de ellos, con una finalidad exploratoria del territorio se procedió a la revisión y análisis tanto, de la bibliografía sobre franquicias como sobre comunicación y NTIC aplicada a las mismas. 
El segundo tipo de observación documental realizado, paralelo en el tiempo, consistió en la búsqueda heurística en publicaciones periódicas de carácter técnico-profesional. Con esta búsqueda se pretendió conocer con mayor exactitud las características concretas de este sistema comercial y su interrelación con las NTIC.

\subsection{Análisis Cualitativo- Panel de Expertos}

Mientras que la encuesta sería el paradigma de la metodología cuantitativa, la discusión de grupo lo será de la cualitativa. Ambas perspectivas metodológicas se complementan. Por ello, para obtener unos resultados completos, se han combinado ambas técnicas, comenzando por la dinámica de grupo en la que participaron expertos implicados de las dos áreas estudiadas.

Dicha reunión se celebró en la Edificio de Ciencias Sociales de la Universidad de Alicante en marzo de 2009, y participaron expertos y profesionales en franquicia, Nuevas Tecnologías, Comunicación, Publicidad y Relaciones Públicas.

Uno de los principales objetivos de la reunión era el de analizar y validar el cuestionario que una vez probado y aprobado por todos los asistentes a modo de pre-test, se les envió por correo electrónico. Tras este primer borrador, los expertos realizaron enmiendas y comentarios, que una vez considerados por los investigadores dieron paso a la siguiente etapa de la investigación: la encuesta.

\subsection{Análisis Cuantitativo: Cuestionario on-line}

En nuestro caso, se han utilizado técnicas de investigación cuantitativas con el objetivo de acercarse a la realidad de gestión de NTIC de un determinado número de empresas franquiciadoras. La información a recoger sería de las empresas franquiciadoras previamente determinadas en función de un criterio que no era otro que su ubicación geográfica y su inscripción en el registro Oficial de Franquiciadores del Ministerio de Industria, Turismo y Comercio a fecha de febrero de 2009. El universo de estudio se compuso de un total de 127 empresas.

Recientemente ha aparecido la encuesta on-line, que permite contestar las preguntas desde cualquier ordenador conectado a Internet, permitiendo una gran comodidad al encuestado y un mejor almacenaje de datos al encuestador para su posterior análisis. En un espacio web proporcionado por la Universidad de Alicante, se colgó el cuestionario definitivo.

El diseño del cuestionario se estructuró en cuatro partes: una primera de cuestiones generales sobre la empresa encuestada, la segunda parte contiene preguntas sobre la comunicación que la empresa lleva a cabo para sus franquiciados actuales. La tercera parte contemplaría las NTIC desarrollas para los potenciales franquiciados de la cadena, y por último, una cuarta parte que pregunta sobre las NTIC Ilevadas a cabo con los clientes o consumidores finales de la red de establecimientos. 
El cuestionario posee un total de 59 ítems estructurados en los cuatro bloques presentados. De entre las preguntas formuladas, la inmensa mayoría son cerradas, la persona entrevistada no podía salirse de unas categorías impuestas en el cuestionario. Sólo si su respuesta era no, la pregunta se convertiría en abierta en algunos casos, al solicitarle en este caso que argumentase el motivo por el cual respondía no.

\subsection{Revisión on-line}

Una vez obtenidas las respuestas de las empresas franquiciadoras y antes de comenzar a realizar los análisis estadísticos y los cruces de variables, se decidió realizar una pequeña revisión on-line de la web de las empresas participantes. De este modo, se comprobó que las respuestas facilitadas se correspondían con la realidad.

\section{Desarrollo de la Investigación}

La presente investigación se acometió durante el año 2009. A partir del mes de enero se definieron los objetivos a alcanzar y la muestra de estudio a analizar. Una vez determinados los objetivos de la investigación se planteó la necesidad de, mediante un panel de expertos, crear la herramienta de análisis válida.

El enlace del cuestionario se hizo llegar a los encuestados desde el mes de mayo de 2009 a través de correo electrónico. Una vez superado el periodo de respuesta (noviembre 2009) se obtuvo un nivel de respuesta del 23,62\% sobre el total del universo, obteniendo un margen de error de $13 \%$ si consideramos un nivel de confianza del 90\%, una proporción esperada del $50 \%$ y un efecto de diseño de 1.

\section{Resultados}

Se presentan en primer lugar los resultados globales para, a continuación, detallarlos por sectores de actividad.

\subsection{Organización general de comunicación}

En cuanto al presupuesto dedicado a acciones de comunicación y publicidad, ninguna empresa dedicó menos de $1.000 €$ anuales a comunicación, encontrando la mayor aglutinación de empresas en el intervalo existente entre 100.001 y $500.000 €$ anuales, en concreto un $23,3 \%$. El $20 \%$ de las empresas destinan a comunicación publicitaria entre 6.001 euro y 12.000 euros.

Respecto a la inversión dedicada a la comunicación por Internet o publicidad on-line, los resultados obtenidos indican que la mayoría (el 44\% de las franquicias) tan sólo destinaron a esta partida presupuestaria un $10 \%$ de su inversión en comunicación, o menos. Son significativas las cifras que reflejan que el $20 \%$ de las empresas dedicaron un $20 \%$ a comunicación on-line, y por último el $17 \%$ de las empresas dedicó el $60 \%$ más de su presupuesto publicitario, al medio on-line. 
Cruzando este último dato con el anterior, obtenemos que las empresas que otorgan más peso a la comunicación en las NTIC son las que menos dinero gastan en comunicación. De esta manera, el $75 \%$ de las empresas que invierten en publicidad de 1.000 a 6.000 euros, destinan más del $60 \%$ de ese dinero a comunicación a través de las Nuevas Tecnologías. Por otro lado, las que más invierten, son las que más gastan en medios off-line, ya que el $100 \%$ de las enseñas que gastan más de $500.000 €$ en comunicación invierte un $10 \%$ de ese presupuesto en las NTIC.

Internet es un medio que permite maximizar la inversión en comunicación, siendo idóneo para presupuestos bajos. Esto se corresponde con la tendencia observada en el estudio y muestra que las enseñas que tienen un presupuesto más humilde son conscientes de esta realidad, aunque también deberían sumarse las enseñas que no alcanzan los $12.000 €$ o los $25.000 €$ (ya que dedican a las NTIC un $30 \%$ y un $17,5 \%$ respectivamente).

Además del presupuesto destinado a comunicación, puede haber otros gastos internos originados por el uso de las NTIC, como por ejemplo el personal que la propia empresa destine a esos menesteres.

Otro de los resultados destacables de la presente investigación es que la mayoría de enseñas tiene una persona o departamento en su propia estructura dedicado a las NTIC. Así, el $53,3 \%$ de las franquicias posee una persona/departamento dentro de la empresa dedicados a la comunicación on-line.

Hay un $40 \%$ de empresas que delegan totalmente su comunicación on-line a empresas externas, pero eso no significa que sean las únicas que subcontratan algunos aspectos relacionados con las NTIC. El 53\% de las empresas que tienen empleados propios trabajando en las nuevas tecnologías, también subcontratan este tipo de servicio. De esta manera, el $70 \%$ de las empresas subcontrata servicios de NTIC. Sólo un 3,3\% manifiesta poseer un departamento integrado por varias personas dedicado a la comunicación on-line.

La naturaleza del tipo de empresas a las que las redes franquiciadoras confían su comunicación on-line es mayoritariamente una empresa especializada en este tipo de comunicación ( $60 \%$ de los casos), dejando de lado a la tradicional empresa de publicidad de servicios plenos o generalista (sólo el $10 \%$ de los franquiciadores confían en ellas).

\subsection{Comunicación con los franquiciados actuales}

Un de las principales herramientas de comunicación interna en este tipo de empresas es el boletín interno o newsletter de franquicia.

Tan sólo el $50 \%$ de las redes analizadas utilizan este valioso instrumento de información interna. La totalidad de las enseñas que lo desarrollan lo hace a través de medios digitales (e-mail, newsletter, web o Intranet). Es decir, el $100 \%$ de los boletines internos son en formato electrónico, olvidando totalmente el soporte papel como una posibilidad para este 
tipo de comunicaciones. Además, la valoración general de los usuarios de este tipo de herramientas es positiva.

El uso de este boletín está implantado sólo en la mitad de la enseñas franquiciadoras, siendo la frecuencia de uso también escasa. De esta manera, tan sólo un 6,7\% realizan un envío semanal, la misma cantidad que los que lo realizan anualmente. Las empresas que prefieren realizarlo mensualmente son un 20\%. La frecuencia más común en estas empresas para la distribución de boletines internos es cada tres meses, con un 33,3\%. El mismo porcentaje, un $33 \%$ de las empresas, no ha determinado una periodicidad fija en sus envíos, por tanto no están planificando el uso de esta herramienta.

La otra gran herramienta de las NTIC para la comunicación interna es la Intranet. Una herramienta con más utilidades y posibilidades que la anterior. Las redes franquiciadoras son conscientes de ello y han implantado esta tecnología en mayor proporción. Un 66,7\% de las enseñas analizadas la posee y, además, valora muy positivamente.

De ese $66,7 \%$ que tiene Intranet, el 25\% también utiliza redes sociales para su comunicación, considerando esos métodos muy efectivos en un $20 \%$ de las ocasiones, efectivos en un $40 \%$ y poco efectivos en un $40 \%$. El $100 \%$ de las empresas que utilizan las redes sociales sin tener Intranet las consideran efectivas.

Si se cruza la variable Intranet con newsletter obtenemos que el $86,7 \%$ de las empresas que utilizan el newsletter, también disponen de Intranet.

En cuanto al tiempo que lleva esa Intranet funcionando dentro de la empresa, el 50\% de las empresas la tienen en marcha desde hace entre 2 y 4 años, el $30 \%$ desde hace 5 ó más años, y el 20\% comenzó a usarla en 2009.

Es de gran importancia conocer las utilidades que posee esa Intranet y, para las empresas que han participado en el proyecto, son las siguientes:

Comunicación entre franquiciados: 60\%

Información de novedades: 85\%

Sugerencias de franquiciados: $58 \%$

Repositorio de documentos:85\%

Pagos: $10 \%$

Peididos: $65 \%$

No existe una uniformidad en el uso de la Intranet pero es significativo que un $40 \%$ de las empresas que tienen Intranet (lo que supondría un $60 \%$ sobre el total de enseñas) no la utilicen como canal de comunicación entre sus franquiciados. De estos datos se extrae que las Intranet aun están en fase de crecimiento. Y aunque tienen una implantación considerable con una más que correcta aceptación, tampoco están aprovechando todas sus oportunidades. 


\subsection{Comunicación con los posibles franquiciados}

En la captación de nuevos franquiciados, las NTIC adquieren una importancia significativa frente a los medios convencionales, al poder ser utilizadas como medio de captación con un coste mucho más reducido.

Así, un $70 \%$ de las empresas franquiciadoras de utilizan los portales web especializados en este sistema de comercialización como vía de captación de clientes. Este medio es, además, el mejor valorado en cuanto a efectividad.

En cuanto al uso de otros soportes en las NTIC para captar nuevos franquiciados, tan sólo encontramos un $20 \%$ que utiliza las webs de diarios on-line como soporte, y además no valoran esa estrategia tan positivamente como el portal especializado. Cabe tener en cuenta que el $83,3 \%$ de las redes que insertan publicidad para captar franquiciados en las webs de prensa, también lo hacen en portales especializados.

Pero la herramienta de las NTIC más utilizada para comunicarse con posibles franquiciados es la propia web de la enseña, ya que el $90 \%$ de las redes franquiciadoras utilizan ese espacio en Internet para informar sobre la posibilidad de adherirse a la central de franquicia.

La inmensa mayoría de las enseñas (90\%) disponen de un apartado en su web dirigido a empresarios interesados en su modelo de negocio, pero no todas las empresas dotan a ese espacio de toda la información que sería necesaria para el futuro asociado. De esta manera, sólo el $62,9 \%$ de las empresas que utilizan este canal presentan un formulario específico de solicitud de información, y un 55,5\% ofrecen digitalmente un catálogo o dossier de franquicia para tener una información más completa. Más representativo es el escaso 18,5\%, que informa de aspectos legales de esa concesión de la franquicia.

Por tanto, aunque mayoritariamente las enseñas franquiciadoras analizadas aprovechan este canal para captar posibles franquiciados, no están explotando todas las posibilidades que, de forma muy económica y efectiva, ofrece.

\subsection{Comunicación con el consumidor final}

Las NTIC, a pesar de su relativa juventud, se deberían englobar dentro de las estrategias de Marketing y Comunicación de la empresa. Hemos ido observando que ya se han ganado un puesto importante dentro del Marketing mix de la empresa franquiciadora, siendo el medio más utilizado. Aunque, en muchas ocasiones se le dedica una parte mínima del presupuesto.

Estudiemos ahora las webs de las empresas franquiciadoras como la principal herramienta de las NTIC para dirigirse al público final.

La primera afirmación es rotunda: el 100\% de las centrales franquiciadoras analizadas tienen una página web propia a disposición de sus clientes finales. Así se demuestra a través de la encuesta realizada y la revisión on-line llevada a cabo por los investigadores. 
En cuanto a los apartados cuya presencia es recomendable en la web, siguiendo el criterio de la reunión de expertos y de las conclusiones extraídas de la revisión bibliográfica se analizaron las siguientes variables, que tras la encuesta, nos ofrecen estos datos:

\section{Apartados dentro de la web de la enseña}

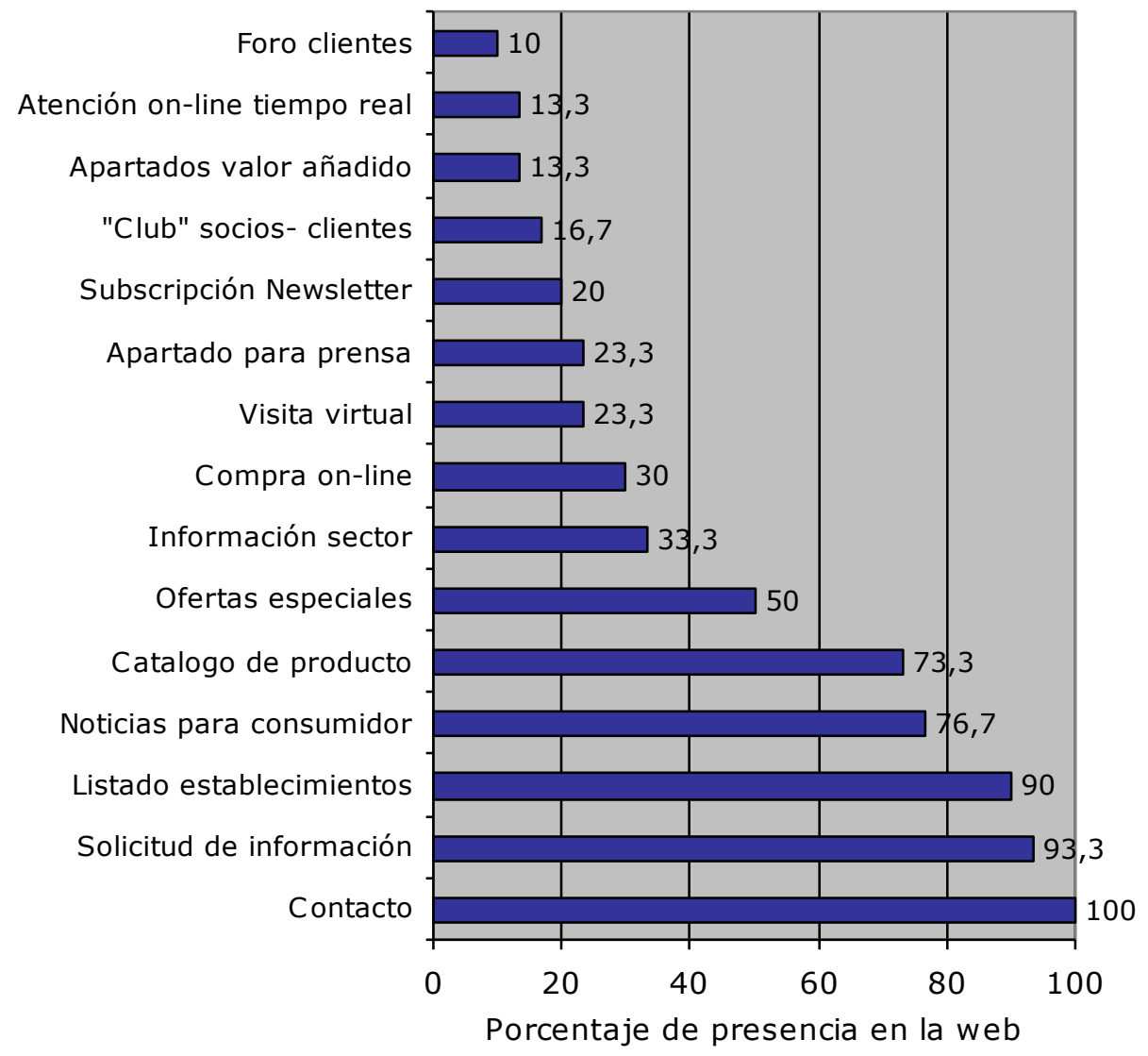

Gráfico 1. Elaboración propia.

Preguntamos por la presencia de un foro para los clientes, para saber (además de la importancia que da la empresa a la comunicación directa con sus clientes), el grado de interacción de esa web y, por tanto, cómo se adapta esa página a la llamada web 2.0.

El porcentaje de empresas que posee este espacio (24\%) junto con el escaso número de franquicias que posee sección de atención en tiempo real, demostraría la siguiente afirmación: las webs de las empresas franquiciadoras presentan un bajo grado de interacción y de adaptación a la web 2.0.

Es de destacar el escaso 13,3\% de webs que ofrece algún apartado de valor añadido, como juegos, descargas, etc. Pero este hecho se corresponde más que con el de la interactividad de la web, con el de fidelización al cliente junto con la sección a modo de club para clientes y la suscripción a una newsletter, ambas también con unos resultados muy bajos. 
Englobamos esas tres secciones dentro de la idea de fidelización al cliente, ya que esos tres apartados van encaminados a crear un sentimiento de pertenencia a la marca y crear lazos más fuertes entre empresa-cliente.

La gran ausencia de estos apartados en las webs contrasta con los resultados obtenidos tras plantear a los franquiciadores si desarrollan acciones tendentes a fidelizar a los clientes. El $87 \%$ manifiesta que sí, pero tan sólo un $16,7 \%$ dedica un apartado en la web a este fin.

Otro gran contraste es el que se da entre el número de enseñas que afirman recoger los datos de correo electrónico con el fin de comunicarse con clientes por ese medio y el número de webs que ofrecen la posibilidad de suscribirse a una newsletter. Mientras que el 63,3\% de las empresas afirma que recoge los datos, tan sólo un $20 \%$ ofrece la subscrición a newsletter.

Siguiendo con el repaso de las secciones, vemos que sólo un 23,3\% ofrece a los medios de comunicación un apartado exclusivo para el acceso de los mismos, facilitándoles toda la información que les pueda interesar, así como logotipos, imágenes o videos. Otra carencia importante de las webs es la posibilidad de compra on-line. Sólo un $30 \%$ ofrece esa posibilidad.

Respecto a la frecuencia con la que las enseñas franquiciadoras actualizan los contenidos de su web, en el $46,7 \%$ de los casos, pasa un mes o más desde la última actualización. En algunos casos (7\%) Ilama la atención las empresas dejen pasar más de seis meses. De esta forma, se explica lo analizado con anterioridad. Si no se actualiza la web, es imposible comunicar promociones a través de ella, y mucho más crear un vínculo a través de este canal con sus visitantes, imposibilitando la creación de comunidades o de foros de comunicación.

La lentitud en la actualización de contenidos contrasta con los datos ofrecidos con anterioridad, donde se podía comprobar que el $56,6 \%$ de las empresas franquiciadoras tienen una persona o un departamento responsable de la web o la comunicación on-line. Estos plazos tan elevados para actualizar la web demuestran un estatismo lejano a lo pretendido con la web 2.0 y a la actual evolución de Internet.

\subsection{Resultados por sectores}

Consideramos interesante ofrecer los resultados en relación a los tres principales sectores de actividad diferenciados por las principales consultoras en franquicia: servicios, retail y hostelería.

Esquematizando los resultados, se concluye que las empresas franquiciadoras pertenecientes al sector Servicios: 
Realizan una menor inversión en comunicación.

Dedican un mayor porcentaje a comunicación on-line dentro del total invertido en comunicación.

Cuentan con una mayor implantación de Intranets. (el 78,6\% frente al $66,7 \%$ general)

Se publicitan más en otras webs (el 71,5\% frente al 50\% general).

Cuidan menos los contenidos de sus webs, ya que:

- Sus webs son las que más estatismo y menos interactividad presentan, alejándose de la web 2.0.

- Sus webs son las que menos utilizan herramientas de fidelización y valor añadido.

- Sus webs son las que menos aprovechan la oportunidad de comunicación con la prensa.

Las empresas franquiciadoras pertenecientes al sector Retail:

Realizan más inversión en comunicación.

Del total invertido en comunicación dedican el menor porcentaje a comunicación on-line.

Cuentan con una menor implantación de Intranets (un 50\% frente al $66,7 \%)$.

Se publicitan menos en otras webs (sobre todo especializadas).

Son las que más utilizan su propia web como canal de comunicación con el posible franquiciado.

Utilizan su web, en gran medida, para presentar ofertas especiales.

Sus webs son las que presentan en mayor medida herramientas de fidelización y valor añadido.

Debido al bajo número de enseñas del sector Hostelería que ha participado en este estudio, tan sólo se presentan algunos datos significativos referentes a este sector:

El $100 \%$ de las empresas posee un presupuesto entre 25.000 y $100.000 €$.

El $100 \%$ de los encuestados contrata a empresas especializadas en comunicación interactiva.

El $100 \%$ de los encuestados utiliza portales especializados en franquicias para captar franquiciados.

El $100 \%$ utilizan su web como canal de comunicación con posibles franquiciados.

\section{Conclusiones.}

Respecto a la inversión en comunicación, el 60\% de las redes franquiciadoras invirtió menos de $50.000 €$ anuales, siendo éstas en su mayoría, del sector servicios. Las que más invierten son del sector retail. De esa inversión, como se ha analizado antes el porcentaje dedicado a 
comunicación a través de NTIC es reducido, sucediendo la inversa del caso anterior: los que más dedican a nuevas tecnologías pertenecen al sector servicios (y a pequeñas redes) y los que menos al sector retail ( $y$ a grandes redes).

Se llega a la conclusión, pues, que las empresas franquiciadoras que más invierten en comunicación son las que menor porcentaje dedican a las NTIC, y viceversa.

Más de la mitad de las enseñas tiene a una persona o equipo responsable de las NTIC dentro de la empresa, aun así, el $70 \%$ subcontrata este tipo de comunicación. De entre las empresas que lo subcontratan, el 85,7\% confía en empresas especializadas en comunicación on-line, y otro $15,3 \%$ sigue delegando este tipo de comunicación a agencias de servicios plenos, y casualmente éstas son empresas consideradas grandes redes franquiciadoras.

Respecto a la comunicación interna, sólo la mitad de las empresas utiliza boletines internos. Además, los envíos presentan una frecuencia muy espaciada en el tiempo y, muchas veces, aleatoria. Aunque el $100 \%$ de los que realiza este tipo de boletines lo hacen en formato electrónico (habiendo relegado totalmente el papel), el uso de esta herramienta tan económica y efectiva se debería desarrollar en mayor medida en las centrales franquiciadoras.

El $86,7 \%$ de esas empresas que realizan boletines internos posee Intranet, lo cual es significativo, ya que la media de uso de estas redes internas es del $66,7 \%$. Pero la mayoría de esas Intranets no permiten la comunicación entre sí a los franquiciados ni entre los franquiciados y la central, dos utilidades de gran potencial. El estudio determina que el uso de Intranets en las franquicias analizadas está en fase de crecimiento.

El uso de las NTIC para captar posibles franquiciados es masivo, ya que es el medio de comunicación más utilizado con el fin de captar emprendedores es Internet. Y más concretamente, el soporte de portales web especializados en franquicia (principalmente de consultoras), con un 70\%, siendo además la acción mejor valorada en cuanto a utilidad. A pesar de este alto porcentaje, sólo un $20 \%$ confía en otro tipo de soporte on-line (prensa multimedia) para lograr este fin.

En cuanto al uso de la propia web para captar franquiciados, el $90 \%$ de las empresas dedican un apartado exclusivo para ello, pero tampoco lo utiliza aprovechando todo su potencial. Sólo un $55,5 \%$ de los que tiene esa sección pone a disposición del interesado un dossier de información, un $18,5 \%$ expone las condiciones legales y un $62,9 \%$ incluye un formulario o solicita información de contacto a este fin.

Para finalizar, en la gestión de la comunicación dirigida al consumidor final, las NTIC tienen mucho peso en la elección de medios, ya que el $70 \%$ de las enseñas(el porcentaje más alto entre las acciones de comunicación realizadas), utiliza estas tecnologías. 
También es significativo el porcentaje de enseñas con web propia, el $100 \%$, lo cual sería una magnífica señal si no fuese porque se trata en su mayoría de sites anclados en el estatismo y la carencia de interactividad de la web 1.0, sobre todo las del sector servicios y las grandes redes, alejándose de las posibilidades que ofrece la web 2.0.

Además, aunque el $86,7 \%$ de las enseñas está muy interesadas en fidelizar a sus clientes, tan sólo el $16,7 \%$ de las webs presentan secciones y herramientas para conseguirlo, en su mayoría del sector retail.

Sólo el 23,3\% dedica un apartado exclusivo para prensa, dónde los medios informativos puedan encontrar todo el material necesario sobre la enseña. Tampoco utilizan el potencial de la web para trasladar o crear ofertas comerciales. Y, siguiendo con el comercio, sólo el $30 \%$ ofrece la opción de compra on-line, siendo ese porcentaje aun menor en las redes de gran tamaño.

En clave positiva, destacamos el incipiente uso de las redes sociales entre las enseñas. Pese a que en 2009 todavía no habían alcanzado el grado de popularidad actual, las franquicias ya comenzaban tímidamente a integrarse en ellas.

Las NTIC están planificadas dentro de los obejtivos y estrategias de la empresa pero su implantación no es óptima. Los anunciantes son conscientes de su importancia pero no las desarrollan, aplican e implementan correctamente.

La investigación concluye que las empresas franquiciadoras no aprovechan correctamente todas las oportunidades que las NTIC les ofrecen para cumplir de forma eficaz y eficiente sus diversos objetivos de comunicación. 


\section{REFERENCIAS BIBLIOGRÁFICAS}

ALLEN, R. L. (1994). "Franchisor-Franchisee: Communication Maintainsthe Marriage", Nation's Restaurant News, 38, 27. En http://findarticles.com/p/articles/mi_m3190/is_n12_v28/ai_14919958/ (consultado: 12/02/2011)

ANDERSON, E., y WEITZ, B. (1989). "Determinants of continuity in conventional industrial Channel Dyads" Marketing Science, 8 (4), 310-323. En http://www.jstor.org/stable/183978

(consultado: 01/02/2011)

ANDERSON, J.C., y NARUS, J.A. (1984). "A model of the distributor's perspective of distributor-manufacturer working relationships", Journal of Marketing, 48, 62-74. En http://www.jstor.org/stable/1251511 (consultado: 01/02/2011)

BAENA, V. (2010). "Teorías y líneas de investigación en el sistema de franquicia: una revisión desde los años 60 hasta 2009". Cuadernos de Gestión, 10(2), 46-66. doi: 10.5295/cdg.100155vb

BOLEA DE ANTA, A. (1988). La empresa de franquicia en el ámbito de la comunicación: Un modelo de aplicación al campo del diseño y moda de España. Tesis Doctoral no publicada, Universidad Complutense de Madrid. Madrid, España.

BOLEA DE ANTA, A. (1990). Los Grandes de la Franquicia. El secreto de su éxito. Madrid, Ediciones Ciencias Sociales.

BUZZI, G. (coord.) (2009). El Modelo de la Nueva Agencia. En http://www.slideshare.net/francisco.goldaracena/el-modelo-de-la-nuevaagencia-2597058 (consultado: 01/03/2011)

CAMPOS FREIRE, F. (coord.). 2010. El cambio mediático. Sevilla-Zamora, España, Ed. Comunicación Social.

CARO, A. (2009). Editorial: "Publicidad y crisis capitalista". Pensar la Publicidad, 3(1), 9-14.En http://revistas.ucm.es/inf/18878598/articulos/PEPU0909120009A.PDF (consultado: 15/02/2011)

DAVIS, P.J. (2004). "Effective communication strategies in a franchise organization. Corporate communications". An International Journal, 9(4), 276-282. doi: 10.1108/13563280410564011

DICKEY, M.H. (2001). Electronic communication channels in franchise organizations. Tesis Doctoral no publicada. Luoisiana State University and Agricultural \& Medical College. 
HERNÁNDEZ, R. (2010). "La publicidad médica como se entendía hace diez años ha muerto". Revista Anuncios, jun.28, 27-28.

KNIGHT, R.M. (1986). "Franchising from the Franchisor and Franchisee Points of View" Journal of Small Business Management, 24, 8-15.

LAIR, D.J. (2006). A rhetorical-cultural analysis of the franchise of 'The Apprentice'. Tesis doctoral no publicada, Universidad de Utah, Salt Lake City.USA.

LAURIE, C. (2000). Partners for the Millennium. Franchising World, 32, 12-20.

MOHR, J.J., FISHER, R.J., y NEVIN, J.R. (1996) "Collaborative communication in interfirm relationships: Moderating effects of integration and control" Journal of Marketing, 60 (3), 103-115. En http://www.jstor.org/stable/1251844 (consultado: 01/02/2011)

MOHR, J.J., y NEVIN, J.R. (1990). "Communication strategies in marketing channels: A theoretical perspective" Journal of Marketing, 54 (4), 36-51. En http://www.jstor.org/stable/1251758 (consultado: 15/02/2011)

MONROY, M.F., y ALZOLA, L.M. (2005). "An analysis of quality management in franchise system". European Journal of Marketing, 39(5/6), 585-605. doi: 10.1108/03090560510590728

MONSERRAT-GAUCHI, J. (2005). Sistemas y procesos de comunicación organizacional en las empresas franquiciadoras: creación de un modelo de optimización de la eficacia. Tesis doctoral no publicada. UCH-CEU. Valencia, España.

MONSERRAT-GAUCHI， J. (2008). "Análisis comparativo de las estrategias de Comunicación en franquicia. Enseñas consolidadas versus emergentes". Revista Latina de Comunicación Social, 63,106-120. doi:10.4185/RLCS-632008-756-106-120

MUBLEMAN, J. (1996). "The Franchise Relationship Channel". Franchising World, 28 (1), 20-24.

TIKOO, S. (2002). "Franchiser influence strategy use and franchisee dependence and experience". Journal of Retailing, 78(3), 183-192. doi: 10.1016/S00224359(02)00064-7

TORMO, E.A. (2009). Go Franquicia! Madrid, Ed. Selina Olmedo.

WALLOVITS, J., y VIRGILI, P. (2011). La reconciliación con el consumidor. Barcelona, España: Empresa Activa, Ediciones Urano.

[Recibido: 21 de julio de 2011. Aceptado con cambios: 6 de febrero de 2012 . Aceptación final: 7 de marzo de 2012]. 